7 Nakayama Y, Nakanishi N, Hayashi T, et al. Pulmonary artery reflection for differentially diagnosing primary pulmonary hypertension and chronic pulmonary thromboembolism. J Am Coll Cardiol 2001; 38: 214-218.

8 Castelain V, Hervé P, Lecarpentier Y, et al. Pulmonary artery pulse pressure and wave reflection in chronic pulmonary thromboembolism and primary pulmonary hypertension. J Am Coll Cardiol 2001; 37: 1085-1092.

9 Hughes AD, Park C, Davies J, et al. Limitations of augmentation index in the assessment of wave reflection in normotensive healthy individuals. PLoS One 2013; 8: e59371.

10 Huez S, Brimioulle S, Naeije R, et al. Feasibility of routine pulmonary arterial impedance measurements in pulmonary hypertension. Chest 2004; 125: 2121-2128.

11 Murgo JP, Westerhof N. Input impedance of the pulmonary arterial system in normal man. Effects of respiration and comparison to systemic impedance. Circ Res 1984; 54: 666-673.

12 Hughes AD, Parker KH. Forward and backward waves in the arterial system: impedance or wave intensity analysis? Med Biol Eng Comput 2009; 47: 207-210.

\title{
Neutrophil gelatinase-associated lipocalin in idiopathic pulmonary fibrosis
}

\begin{abstract}
To the Editor:
Neutrophil gelatinase-associated lipocalin (NGAL) is a $25 \mathrm{kD}$ lipocalin that is covalently bound to matrix metalloproteinase (MMP)-9 produced by neutrophils [1]. NGAL in blood or bronchoalveolar lavage fluid (BALF) may reflect neutrophilic inflammation in the lungs [2, 3], and it is highly induced in injured epithelial cells, including those in the lung [4]. Possible roles for neutrophilic inflammation and epithelial injury have been reported in idiopathic pulmonary fibrosis (IPF). Thus, we hypothesised that NGAL may be associated with the pathogenesis of IPF. To investigate the roles of NGAL in IPF, we used immunohistochemical staining for lung specimens and measured plasma and BALF NGAL levels. Our study was approved by the Ethics Committee of Kyoto University (approval No. E438), and written informed consent was obtained from all study participants.
\end{abstract}

First, we immunohistochemically stained the lung tissue specimens of six IPF patients, two nonspecific interstitial pneumonia (NSIP) patients, and a control (normal area distant from the lesion of surgically diagnosed organising pneumonia) for NGAL using a conventional method [5]. We also performed sequential immunofluorescent staining for NGAL and MMP-9. Immunohistochemical staining showed that NGAL was abundantly expressed in airway epithelial cells that covered the honeycomb cysts in IPF (fig. 1a and b). Further, histologically normal bronchioles in the fibrotic lesions also exhibited abundant NGAL expression, although apparently normal alveolar walls exhibited little NGAL expression. NGAL was also expressed in macrophages, neutrophils and some alveolar epithelial cells.

In NSIP, NGAL was expressed in macrophages and a small number of alveolar epithelial cells. Airway epithelial cells also showed NGAL expression; however, there were fewer positive cells and their staining intensity was weaker compared with that of the IPF specimens (fig. 1c). The control lung specimen revealed an expression pattern similar to that of NSIP patients (fig. 1d). Sequential immunofluorescent staining indicated that NGAL and MMP-9 were similarly expressed in a patchy pattern along the airway epithelial cells, which covered honeycomb cysts in the lung tissues of IPF (fig. 1e and f).

We also measured plasma and BALF NGAL levels for 36 IPF patients (25 males, 27 smokers, 15 with histological diagnosis) who visited Kyoto University Hospital from October 2007 to April 2012 and underwent bronchoalveolar lavage (BAL). IPF was diagnosed on the basis of the official joint statement on IPF published in 2011 [6]. For disease controls, we also enrolled 26 patients as "non-IPF" interstitial pneumonia group (11 males, 14 smokers). The non-IPF group consists of seven patients who had undergone surgical lung biopsy (SLB) (two had NSIP and five exhibited a pathological pattern of bronchiolocentric interstitial pneumonia) and 19 patients with interstitial pneumonia of unknown cause who did not undergo SLB but exhibited "inconsistent with UIP pattern" on high-resolution computed tomography [6]. At the time of enrolment, none of these patients had been treated for interstitial lung disease.

Blood and BALF sampling and pulmonary function testing were performed at the same time within 7 days of admission. In addition, 31 subjects without lung disease were enrolled as healthy controls for plasma NGAL determination that were matched for age and serum creatinine levels. Plasma NGAL levels and BALF NGAL levels were determined using sandwich ELIZA (Antibody Shop, Gentofte, Denmark). BALF albumin concentrations were measured by turbidimetric immunoassay (Superior-Microalbumin kit; Mitsubishi 

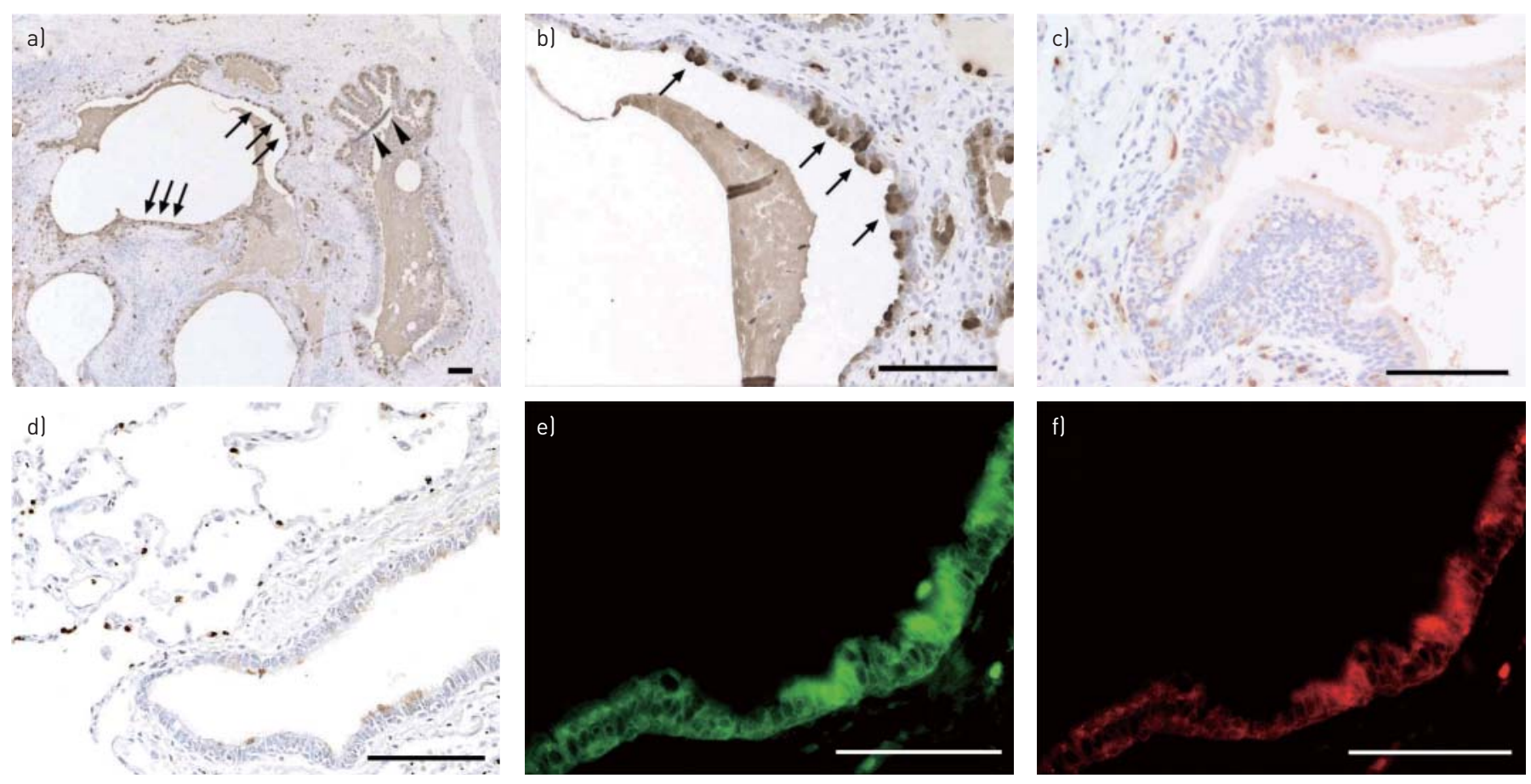

FIGURE 1 Immunohistochemical staining results for neutrophil gelatinase-associated lipocalin (NGAL) in lung specimens from: a and b) idiopathic pulmonary fibrosis (IPF) patients, c) nonspecific interstitial pneumonia (NSIP) patients, and d) a control subject. a) In IPF, NGAL was expressed in those airway epithelial cells that covered the honeycomb cysts (arrow) and histologically normal bronchioles (arrowhead). b) High magnification image of part a). c and d) In NSIP and control specimens, NGAL was expressed in a small number of airway and alveolar epithelial cells and in macrophages. e) and f) Sequential immunofluorescent staining for NGAL and matrix metalloproteinase (MMP)-9 in lung tissues from IPF patients. Both e) NGAL and f) MMP-9 were expressed in those airway epithelial cells that covered the honeycomb cysts. Scale bars $=100 \mu \mathrm{m}$.

Chemical Medience, Tokyo, Japan), and BALF NGAL levels were corrected for BALF albumin concentrations ( $\mathrm{ng} \cdot \mathrm{mg}^{-1}$ albumin). Comparisons of plasma and BALF NGAL levels between groups were made using the Mann-Whitney U-test or Kruskal-Wallis test, $\mathrm{p}<0.05$ was considered significant.

The median (interquartile range) plasma NGAL levels in the IPF patients $\left(55.5(43.3-78.0) \mathrm{ng} \cdot \mathrm{mL}^{-1}\right.$ ) were significantly higher than those in the healthy controls $\left(45.0(36.0-48.9) \mathrm{ng} \cdot \mathrm{mL}^{-1} ; \mathrm{p}<0.001\right)$ and were similar to the non-IPF patients $\left(66.0(55.5-74.3) \mathrm{ng} \cdot \mathrm{mL}^{-1}\right)$. BALF NGAL levels in IPF patients $(481(315-1178)$ $\mathrm{ng} \cdot \mathrm{mg}^{-1}$ albumin) were significantly higher than those in non-IPF patients $\left(229(144-562) \mathrm{ng} \cdot \mathrm{mg}^{-1}\right.$ albumin; $\mathrm{p}=0.03$ ).

In IPF patients, age $\left(r_{s}=0.37, p=0.03\right)$, male sex $\left(r_{s}=0.49, p=0.002\right)$, smoking history $\left(r_{s}=0.39, p=0.02\right)$ and serum creatinine levels $\left(r_{s}=0.61, p<0.001\right)$ were significantly associated with plasma NGAL levels (Spearman's rank correlation). In addition, forced vital capacity $\%$ predicted (FVC \% pred) $\left(\mathrm{r}_{\mathrm{s}}=-0.51\right.$, $\mathrm{p}=0.002)$ and BALF neutrophil percentages $\left(\mathrm{r}_{\mathrm{s}}=0.53, \mathrm{p}=0.001\right)$ were significantly associated with BALF NGAL levels. In the non-IPF group, plasma NGAL levels were significantly associated with blood neutrophil counts $\left(r_{s}=0.40, p=0.04\right)$, but not with other clinical variables. In the same group, BALF NGAL levels were not correlated with either BALF neutrophil percentages or with FVC \% pred.

During a mean follow-up period of 30.8 months (range 7.6-61.4 months), 10 (28\%) IPF patients died. When IPF patients were divided into two groups on the basis of the median BALF NGAL level, patients with higher BALF NGAL levels $\left(>480 \mathrm{ng} \cdot \mathrm{mg}^{-1}\right.$ albumin; $\mathrm{n}=18$ ) had a significantly worse survival compared with those with lower levels $(\mathrm{p}=0.02$ by log-rank test).

It is worth noting that NGAL protein was abundantly expressed in airway epithelial cells that covered the honeycomb cysts in IPF lung specimens. The phenomenon in which air space lumens are covered by bronchial epithelial cells in these honeycomb lesions is called bronchiolisation. In addition, our immunofluorescent staining results indicated that both NGAL and MMP-9 had similar expression patterns in these bronchiolised cells. Previous studies also reported abundant MMP-9 expression in these bronchiolisation areas $[7,8]$. NGAL binds to MMP-9 and exerts a protective effect by preventing MMP-9 degradation [9]. Therefore, along with MMP-9, NGAL may play an important role in alveolar bronchiolisation in IPF. 
In this study, BALF NGAL levels were strongly correlated with BALF neutrophil percentages and FVC \% pred in IPF patients, whereas they were not correlated with either BALF neutrophil percentages or with FVC \% pred in non-IPF patients. This dissimilarity may reflect the difference of neutrophil activity between IPF and other idiopathic interstitial pneumonias. Together with the results of immunohistochemical staining, BALF NGAL may be a surrogate marker that reflects both neutrophilic inflammation and fibrosis severity in IPF. However, a further study with a larger cohort is needed to establish whether BALF NGAL is a prognostic factor independent of disease severity in IPF. Moreover, the clinical utility of the plasma NGAL level as a marker for IPF may be limited. Plasma/serum NGAL has been shown to be a biomarker for acute kidney injury [10]. In the present study, plasma NGAL was strongly affected by renal function rather than by IPF disease severity, and it was not specifically elevated in IPF patients.

Our study had some limitations. First, this was a cross-sectional study with a small sample size. Secondly, the non-IPF group included 19 patients who did not undergo SLB. Therefore, this group could have mistakenly included IPF patients; thus, leading to misclassification. Despite these limitations, this is the first study to demonstrate that NGAL may be involved in the pathogenesis of IPF.

We concluded that NGAL was abundantly expressed in IPF lungs, and that BALF NGAL levels reflected neutrophilic inflammation and disease severity.

0 @ERSpublications

NGAL was abundantly expressed in IPF lungs, and BALF NGAL levels reflected IPF disease severity http://ow.ly/sYTYr

Kohei Ikezoe ${ }^{1}$, Tomohiro Handa ${ }^{1}$, Kiyoshi Mori ${ }^{2}$, Kizuku Watanabe ${ }^{3}$, Kiminobu Tanizawa ${ }^{4}$, Kensaku Aihara ${ }^{5}$, Tatsuaki Tsuruyama ${ }^{6}$, Aya Miyagawa-Hayashino ${ }^{7}$, Akihiko Sokai ${ }^{1}$, Takeshi Kubo ${ }^{8}$, Shigeo Muro ${ }^{1}$, Sonoko Nagai ${ }^{9}$, Toyohiro Hirai ${ }^{1}$, Kazuo $\mathrm{Chin}^{4}$ and Michiaki Mishima ${ }^{1}$

${ }^{1}$ Dept of Respiratory Medicine, Kyoto University Graduate School of Medicine, Kyoto, ${ }^{2}$ Medical Innovation Centre, Kyoto University Graduate School of Medicine, Kyoto, ${ }^{3}$ Dept of Respiratory Medicine, Fukui Red Cross Hospital, Fukui, ${ }^{4}$ Dept of Respiratory Care and Sleep Control Medicine, Kyoto University Graduate School of Medicine, Kyoto, ${ }^{5}$ Dept of Respiratory Medicine, Saiseikai Noe Hospital, Osaka, ${ }^{6}$ Centre for Anatomical Studies, Kyoto University Hospital, Kyoto, ${ }^{7}$ Dept of Diagnostic Pathology, Kyoto University Hospital, Kyoto, ${ }^{8}$ Dept of Diagnostic Imaging and Nuclear Medicine, Kyoto University Graduate School of Medicine, Kyoto, and ${ }^{9}$ Kyoto Central Clinic/Clinical Research Centre, Kyoto, Japan.

Correspondence: T. Handa, Dept of Respiratory Medicine, Kyoto University Graduate School of Medicine, 54 Shogoin Kawaharacho, Sakyo-ku, Kyoto 606-8507, Japan. E-mail: hanta@kuhp.kyoto-u.ac.jp

Received: July 282013 | Accepted after revision: Dec 172013 | First published online: Feb 202014

Conflict of interest: Disclosures can be found alongside the online version of this article at www.erj.ersjournals.com

\section{References}

1 Mishra J, Mori K, Ma Q, et al. Amelioration of ischemic acute renal injury by neutrophil gelatinase-associated lipocalin. J Am Soc Nephrol 2004; 15: 3073-3082.

2 Eagan TM, Damås JK, Ueland T, et al. Neutrophil gelatinase-associated lipocalin: a biomarker in COPD. Chest 2010; 138: 888-895.

3 Betsuyaku T, Nishimura M, Takeyabu K, et al. Neutrophil granule proteins in bronchoalveolar lavage fluid from subjects with subclinical emphysema. Am J Respir Crit Care Med 1999; 159: 1985-1991.

4 Devarajan P. Review: neutrophil gelatinase-associated lipocalin: a troponin-like biomarker for human acute kidney injury. Nephrology (Carlton) 2010; 15: 419-428.

5 Toda Y, Kono K, Abiru H, et al. Application of tyramide signal amplification system to immunohistochemistry: a potent method to localize antigens that are not detectable by ordinary method. Pathol Int 1999; 49: 479-483.

6 Raghu G, Collard HR, Egan JJ, et al. An official ATS/ERS/JRS/ALAT statement: idiopathic pulmonary fibrosis: evidence-based guidelines for diagnosis and management. Am J Respir Crit Care Med 2011; 183: 788-824.

$7 \quad$ Fukuda Y, Ishizaki M, Kudoh S, et al. Localization of matrix metalloproteinases-1, -2, and -9 and tissue inhibitor of metalloproteinase-2 in interstitial lung diseases. Lab Invest 1998; 78: 687-698.

8 Hayashi T, Stetler-Stevenson WG, Fleming MV, et al. Immunohistochemical study of metalloproteinases and their tissue inhibitors in the lungs of patients with diffuse alveolar damage and idiopathic pulmonary fibrosis. Am $J$ Pathol 1996; 149: 1241-1256.

9 Yan L, Borregaard N, Kjeldsen L, et al. The high molecular weight urinary matrix metalloproteinase (MMP) activity is a complex of gelatinase B/MMP-9 and neutrophil gelatinase-associated lipocalin (NGAL): modulation of MMP-9 activity by NGAL. J Biol Chem 2001; 276: 37258-37265.

10 Haase M, Bellomo R, Devarajan P, et al. Accuracy of neutrophil gelatinase-associated lipocalin (NGAL) in diagnosis and prognosis in acute kidney injury: a systematic review and meta-analysis. Am J Kidney Dis 2009; 54: 1012-1024. 